\title{
RISK TREATMENT TEMPLATES FOR CONFIGURABLE REFERENCE MODELING IN THE CONSTRUCTION INDUSTRY
}

\author{
Wael Sharmak, Sven-Eric Schapke, Raimar J. Scherer \\ Institute of Construction Informatics, Dresden University of Technology, GERMANY \\ \{Wael.Sharmak@mailbox.tu-dresden.de; Sven.Schapke;Raimar.Scherer@tu-dresden.de\}
}

\begin{abstract}
Numerous risks impact construction projects and cause changes in their management plans. Unfortunately, not all of them can be identified in advance. Hence, risk management in construction requires proactive as well as reactive treatment. Among the other risk management tasks, risk treatment requires a sound methodology to rapidly develop concrete change actions and alter the corresponding project management plans. In this paper, process modeling techniques are used to develop configurable treatment templates, which describe how treatment can change the schedule plan. Such templates can contribute in structuring configurable reference models which in turn can be tailored and assembled to form up-to-date project schedules. Risk data as a part of reference repository may serve as means of knowledge management by providing all available risk-related information as response to critical events.
\end{abstract}

\section{INTRODUCTION}

The construction industry is project-based. Each project is a one-off unique venture that is characterized by a user-driven initiation, a multitude of project stakeholders and disciplines as well as integral contracts for works and services. As such, construction projects have always been executed by construction networks, i.e. temporal collaborative networked organizations that bring together the required expertise and resources and share the respective project risks.

For the management of construction activities, traditional project management techniques are used that break down the project into manageable, self-containing subtasks with specific goals. Based on a system engineering approach various separate models of the delivered goods and services are used to support planning, coordination and control of the design and production processes. However, no project ever goes totally as planned. Construction projects are exposed to numerous uncertainties and unforeseeable risks that may result in changes to the initial financial, temporal and functional goals as well as the respective design and construction management plans

Particularly, construction-driven risks such as unexpected weather, soil and groundwater conditions, possible accidents as well as changing public regulations and policies are difficult to assess. Most often they require instant decisions on what 
treatment measures have to be taken as well as on the required adjustments of the current designs and the respective project management plans.

Moreover, there are also sever design-driven risks that result from conflicting interests of the project stakeholders and changing owner requirements as well as design errors and omissions induced by short design times and the uniqueness and complexity of products. Design-driven errors are often recognized at a later stage of the project and often require considerable redesign and rework.

The paper introduces a novel, process-centered view on risk management that focuses on the risk treatment plans in foresight of possible as well as in reaction to already occurred risks. To allow for a more rapid development of the risk treatment plans the research pursues the use of reference processes for risk treatment as described in chapter 2.3. To support the approach risk treatment templates have been developed that constitute the structural foundation for altering the current project schedule in accordance to the required risk treatment tasks as shown in chapter 3.

\section{PROCESS MODELING FOR RISK TREATMENT IN AEC}

Although risks in architecture, engineering and construction (AEC) usually affect several aspects of a structure's design and the construction plans, risk management is (if at all) limited to a few separated project management plans. While cost management and scheduling make projections of the anticipated time and money consumption, there are only insufficient management systems to handle threats from external events and later discovered errors and omissions. Most often ad hoc decisions are made that later result in unintentional changes to the project plans. Hence, there is a need for an extension to risk treatment methodologies that allows for handling commonly emerging and unforeseen risks as well as for projecting the risk treatments effects on the overall project plans.

In our research we pursue a process-centered approach to risk treatment planning in which extended business process models provide for (a) the positioning of the risk within the project process, (b) the identification of interdependent elements in related engineering and management systems as well as (c) the analysis and simulation of risk treatment measures. Moreover, to allow for a rapid development of risk treatment plans reference process models that can be adapted to the current project situation are predefined for all-known project risks.

\subsection{Risk Management}

In corporate and project management literature risk management is usually considered a supporting function. Risks are treated in proactive way by monitoring external threats and internal operations in regard to previously identified and classified risks of high probability and/or high impact. According to the established standards [1,9] such risk management typically involves a series of management tasks such as: the (a) identification, (b) assessment, (c) treatment planning, (d) treatment, (e) monitoring, and (f) documentation of the risks.

However, due to the uniqueness of construction products and the multitude of external influences it is hardly possible to identify all construction risks in early project stages. Moreover, even if a risk is identified, it can be underestimated and thus excluded from the proactive risk treatment group. In addition, there is a 
tendency in the construction industry to leave probable risksand react to them when they have occurred, rather than dealing with them in a [7]. Therefore, an extension to traditional risk management for reactive risk nent is suggested to more accurately handle problems that are detected during 1 User : execution. Figure 1 depicts the two interdependent risk management cycles of pivacive and reactive risk treatment.

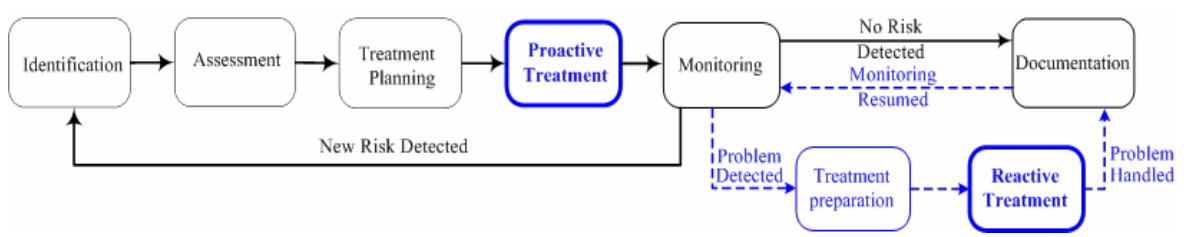

Figure 1 - Proactive and Reactive Treatment Cycles within RM Process.

\subsection{Reference Process Modeling in Construction}

A reference model is a generic conceptual model that formalizes recommended practices for a certain domain. It constitutes a best-practice yet universal description of a class of application domains that can be reused for information systems development in related projects and enterprises [3].

Reference modeling constitutes all activities for constructing and applying reference models. Differences between the existing reference modeling approaches mainly result from the applied technologies for the reuse of the reference models. In respect to the decomposition of the model and the degree of adaptability various forms of reuse from static enterprise-wide recommendations to configurable model modules can be distinguished. In line with several authors in [2] and [3] we distinguish four approaches to reusing reference models:

- Analogical Reuse that imposes no restrictions on the use of reference models.

- Rule-based Configuration in which the reference models comprise explicit rules for their adaptation.

- Generic Configuration in which the possible adaptations of reference models, such as their specialization and instantiation are defined by the methodology.

- Composition that combines multiple reference models in a superordinate one.

A prerequisite for the effective reuse a reference process models is a comprehensive description of its application context. However, despite the importance of process management, integrated process and enterprise models are rarely used in AEC due to the heterogeneity and dynamics of the construction networks. One suitable methodology for modeling collaboration networks from project initiation to workflow definition was proposed in the ArKoS project [6]. Conceptually the modeling architecture comprises three model components:

- Construction Network Model that captures the contextual information of the organizational structures and infrastructures of construction networks,

- Inter-organizational Process Module Chains that represent the goods and service delivery processes on the inter-organizational level.

- Event-driven Process Chains and Workflow Models that detail and finally formalize the processes of the process modules on the task level.

Based on the three modeling components some general solutions to reference process modeling in AEC can be illustrated. Firstly, the construction network model 
provides general context information for the adaptation of reference processes to the current project situation. Secondly, process modeling on the inter-organizational level mainly requires the composition of corporate deliveries in regard to the technical and organizational interfaces of the modules and their pre- and postconditions. Thirdly, the development of the process task models is ideally supported by a comprehensive contextual framework of a construction network and a process module chain. Thus, an effective reuse of reference processes here first of all requires the configuration of process templates to execute the predefined tasks under the given conditions as well as the instantiation of the template parameters.

\subsection{Risk Treatment Planning in Construction}

Central point of reference for the proposed process-centered risk management is the task level representing the detailed design and execution plans. While financial risks can be managed based on subcontractor records, the occurrence of critical events, errors and omissions requires the instant identification of all affected tasks.

The overall goal of the approach is to support the alternation and complementation of the current project plans with predefined reference models for risk treatment called reference risk treatment processes (RTP). Prerequisite for the efficient development of risk treatment plans is a detail model of the current processes. It is assumed that the project processes have been modeled by extended event-driven process chains (eEPCs) [5] to:

- Support Process Planning and Workflow Management: While the eEPC is mainly indented for the integrated modeling of the business processes it also supports construction-specific schedule and resource planning approaches as well as the automatic generation of corresponding workflows [6].

- Support Reuse of Risk Treatment Processes: The eEPC do not only model the technical and organizational context of a task but also allow for representing occurred risks and treatment plans as deviation events and treatment functions. Moreover, it provides for modeling process templates in Configurable EPC (C-EPCs) that extend regular EPCs and allow for defining configuration connectors and configuration functions in reference processes [8].

Figure 2 depicts the overall modeling process of the approach. Overall it comprises a risk effect analysis as well as the selection, configuration and integration of RTPs:

Risk effect analysis: The elicitor of risk treatment planning is the detection of a particular past or future risk. Project management needs to analyze the risks' effects consulting expert knowledge as well as the project schedules identifying all the tasks that can be affected. Moreover, interim goals for the treatment plans shall be defined that must be met under all circumstances.

Selection of Reference Risk Treatment Processes: The RTPs are provided via a reference process catalogue that can be filtered based on the contextual information of the eEPC as well as the risk information and interim goals. The reference catalogue will be developed based on the Process Matrix that already constitutes a collection of over 300 reference processes for AEC in a facet classification [4]. For the management of the RTPs the facets of the process matrix classification will be aligned with eEPCs and extended by risk and performance parameters. Moreover, the reference risk treatment processes will be liked to configurable process structures, such as the risk treatment templates (RTTs) described in chapter 3. 


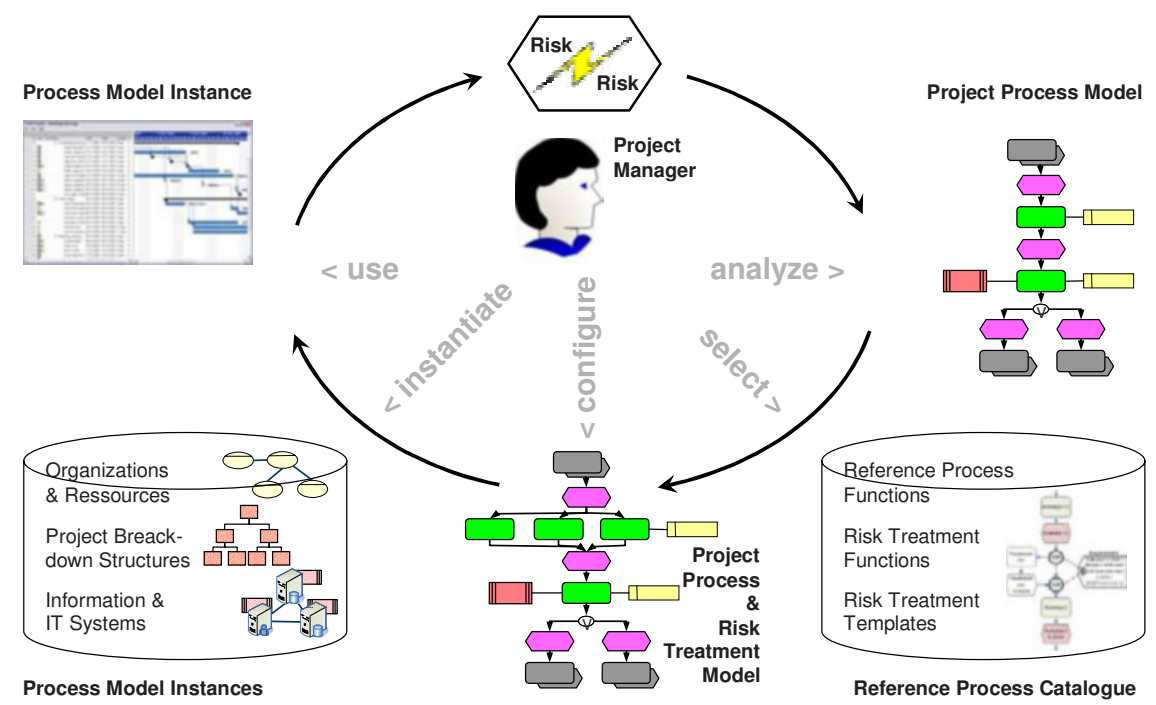

Figure 2 - Process of risk treatment planning.

Configuration of Reference Risk Treatment Processes: The configuration of the RTPs is first of all limited to the configuration functions provided by C-EPCs and formalized in the RTTs. For now it can be assumed that the reference process catalogue contains a RTP for all the affected functions as the original project processes are developed based on the same catalogue. Hence, the biggest challenge of the configuration tasks is to achieve structurally correct processes as demonstrated in chapter 3. However, future research will also have to provide guidance and support for the adaptation of the RTPs to the technological and organizational context of the overall eEPC.

Integration, Instantiation and Usage of Reference Risk Treatment Processes: The integration of the RTPs with the former process plans first of all requires the combination of the affected tasks with the newly developed risk treatment processes. Moreover, until now risk treatment modeling was carried out configuring general process models. For applying the develop process models in project management they have to be transformed to actual project schedules or workflows. This also requires the instantiation of all attributes left unspecified, e.g. assigning values for the names and addresses of responsible persons from the construction network model or start and end times from the overall project process.

\section{TEMPLATES FOR RISK TREATMENT MODELING}

Based on an analysis of possible activity sequences, six risk treatment templates have been developed that represent general types of process changes caused by risks. The risk treatment template can be considered a case-oriented configurable process model that describes a set of possible risk-related sequence change scenarios. After the configuration of the template only one of these scenarios will be used to represent an "actual or virtual" schedule change caused by a specific risk event. 


\subsection{Suggested Risk Treatment Templates}

In accordance to the risk management cycles presented in chapter 2, risk treatment templates are classified into proactive and reactive risk treatment templates. In all templates, the expressions "Risk=ON, Risk=OFF" are used in the if-then statements to indicate the current status of a risk. Risk $O N$ in proactive treatment means that the mentioned risk has approved probability/impact exceeds the agreed threshold. In reactive treatment it means that the risk has evolved to a real problem and the required changes have been approved. Risk OFF indicates the opposite.

In the templates the actual treatment of each risk is defined by a function named "treatment". This function as hierarchical one can be refined into another EPC, which shows the details of the needed actions to handle one specific risk-activity case. Moreover, according to task dependencies, risk treatment can cause cascade action, which means that a treatment in one activity may create problems in logically related activities. Therefore, other actions may be needed to be done to some interrelated activities, e.g. durations and dates adjusting or resources leveling, which is not included in our work.

\subsection{Proactive Risk Treatment Templates}

Insertion Case: In this template, see Figure (3-1), the treatment is done before the risk evolves to a real problem. When it will be found that the risk event has a considerable probability/impact on the targeted activities according to the agreed tolerance thresholds, then treatment function will be included in the schedule plan. The needed resources will be allocated and the needed cost and time will be considered in the project management plans.

Substitution Case: As a countermeasure to the coming danger, certain activities may need to be substituted with other activities which are more suited to the probable new case. In the template shown in Figure (3-2), function (n) is substituted with function $(\mathrm{m})$ to react/avoid/mitigate the highly expected high risk impact. This alternative function, function $(\mathrm{m})$, was not preferred in normal cases because of, e.g. its higher cost, its longer duration, or its complex execution technology etc.

Function Cancelation Case: This template, Figure (3-3) can be used for example in the case when a threat becomes highly expected, so some changes must be done as a response to the coming danger by canceling some planned tasks and adding other new tasks somewhere else in the project schedule.

Parallelism Case: The treatment will be done in parallel with some planned tasks, Figure (3-4). The configurable $\mathrm{OR}$ is used and a requirement will limit its configured scenarios to two models only; (1) AND when Risk=ON, (2) normal sequence when Risk= off.

\subsection{Reactive Risk Treatment Templates}

In reactive templates, risk event will interrupt and stop temporarily the execution of a function, and four important elements will appear between the interrupted function and its following event: risk event, Treatment preparation and Treatment functions, and Resuming function. The developed interruptive treatment models are: 


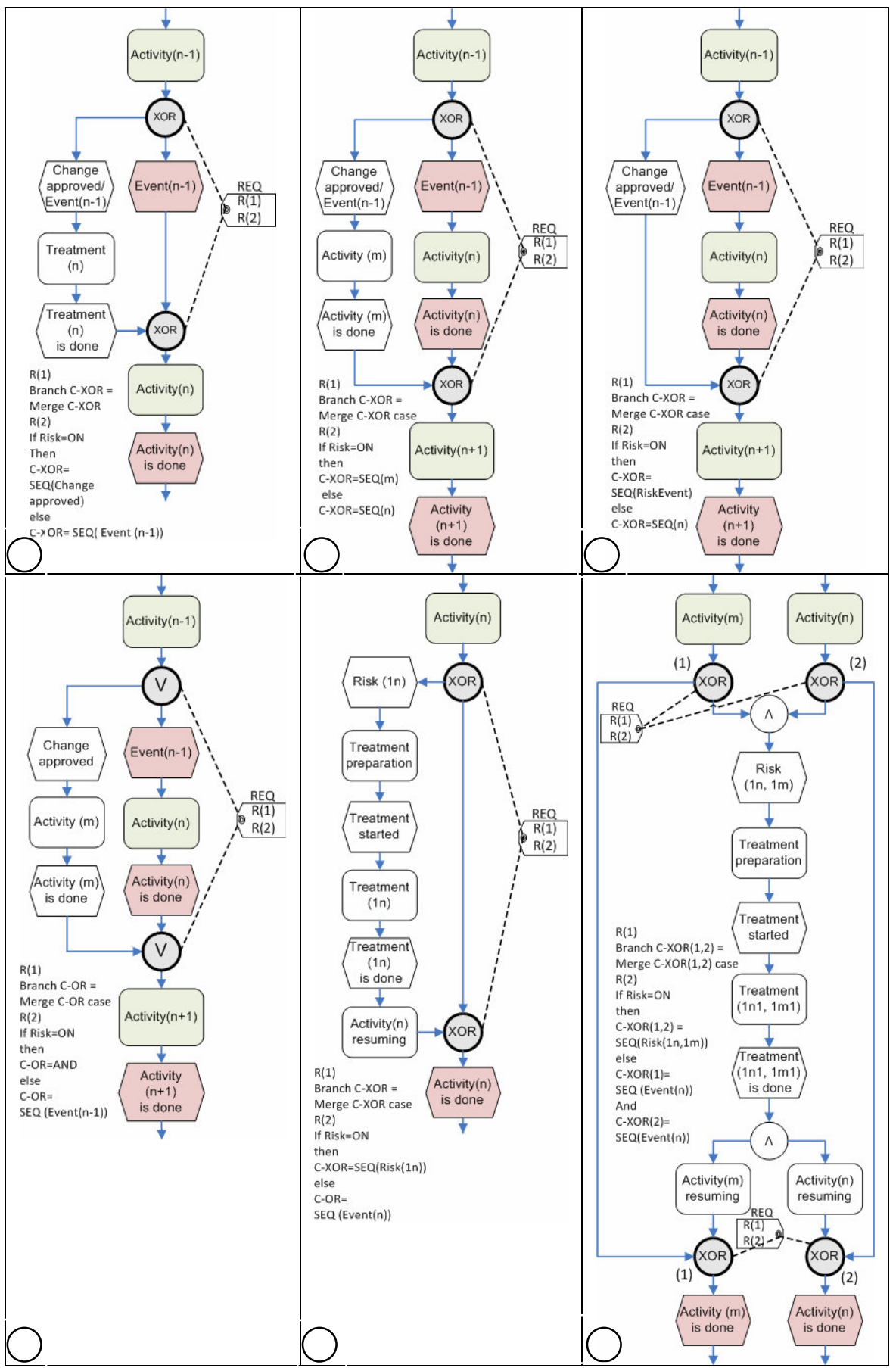

Figure 3 - Proactive and Reactive Risk Treatment Templates 
Treatment Case: Risk (1n) interrupted Function (n), therefore Treatment (1n) is needed to handle the risk, after that Function (n) can be resumed, see Figure (3-5). Stop case is a special case of the treatment one. The risk event will cause a stop in this function until the risk bad effect is finished. This case represents the negative reaction to the risk, e.g. the case of unexpected bad weather which will stop the work in the outdoor activities until the weather becomes better.

Parallel Activities Case: In the case that a risk will interrupt some parallel tasks, all affected parallel paths will need to be merged in one path, on which the treatment of this risk will take place. After the treatment is done/the risk effect is finished, the path can be splitted again to the same old paths, the interrupted tasks will be resumed and after that the successor planned tasks will be executed in parallel as planned, see Figure (3-6).

\section{CONCLUSION}

The paper introduces a novel view on risk management that focuses on the risk treatment plans in foresight of possible as well as in reaction to already occurred risks. To support the development of the risk treatment plans a catalogue of reference risk treatment processes is pursued. Six risk treatment templates have been introduced that constitute the structural foundations for developing the reference risk treatment processes. Each template is a configurable branch which can be linked to the schedule network using configurable connectors. Configurable connectors can be adapted to include or exclude the exceptional path. Each template illustrates one way of risk-caused change in the project schedule. However each risk can cause more than one change so more than one template may be needed. Such template will not provide how to treat a risk, as it is only an activity-sequence description which can suit many kinds of risk situations. Therefore, joins to reference data repository are needed to provide all related Risk-Task information. The structure of the needed database, the ontology description of the suggested task reference model and the assembling method of the adapted models will be the next steps of this work.

\section{REFERENCES}

1. AS/NZS 4360: Risk Management Standards, Australia, 1999.

2. Becker J., Knackstedt R. (Eds.) Referenzmodellierung 2002. Methoden - Modelle - Erfahrungen. Münster 2002.

3. Fettke P, Loos P, (Eds). Reference Modeling for Business Systems Analysis. Idea Group Publishing, 2007.

4. Katranuschkov P., Gehre A., Scherer R.J., Wix J. \& Liebich T. (2004): User Requirements Capture in Distributed Project Environments: A Process-Centred Approach. In: Proceedings of Xth International Conference on Computng in Civil and Building Engineering (ICCCBE), Weimar, Germany, 2004.

5. Keller G, Nüttgens M, Scheer A-W. Semantische Prozessmodellierung auf der Grundlage,, Ereignisgesteuerte Prozessketten (EPK)“, 1991.

6. Loos P., Vanderhaeghen D. (Eds.) Kollaboratives Prozessmanagement: Unterstützung kooperationsund koordinationsintensiver Geschäftsprozesse am Beispiel des Bauwesens, Logos Berlin, 2007.

7. Loosemore, Martin. Risk Management in Projects. Taylor \& Francis Group, London, 2005.

8. Mendling J, Recker J, Rosemann M, van der Aalst W. Generating Correct EPCs from Configured CEPCs. In: Proceedings of the 21st Annual ACM Symposium on Applied Computing. France, 2006.

9. PMBOK Guide. A Guide to the Project Management Body of Knowledge, Project Management Institute, Pennsylvania, USA, 2004. 